\title{
Knowledge space theory, formal concept analysis, and computerized psychological assessment
}

\author{
Andrea Spoto, Luca Stefanutti, and Giulio Vidotto \\ University of Padua, Padua, Italy
}

\begin{abstract}
In the present study, the use of knowledge space theory (KST), jointly with formal concept analysis (FCA), is proposed for developing a formal representation of the relations between the items of a questionnaire and a set of psychodiagnostic criteria. This formal representation can be used to develop an efficient adaptive tool for psychological assessment. Rusch and Wille (1996) have shown some interesting connections between KST and FCA; these connections are applied in the construction of knowledge structures, starting from a formal context representing the relations between items and criteria. The proposed general methodology was applied, as an example, to the Maudsley Obsessional-Compulsive Questionnaire. We used a data set provided by a sample of patients with a diagnosis of obsessive-compulsive disorder to validate the obtained structures. The parameters of the basic local independence model (BLIM) were estimated for the obtained knowledge structures. The fit of each model was tested by parametric bootstrap because of the sparseness of the derived data matrix. The results are discussed in light of both psychological and methodological relapses. In particular, we propose a reinterpretation of the BLIM parameters that seems suitable for testing reliability and construct validity; furthermore, it is pointed out how the obtained structures could represent the starting point for the development of a computerized assessment tool.
\end{abstract}

In the present study, knowledge space theory (KST) is applied, jointly with formal concept analysis (FCA), in the definition of a methodology for constructing a formal representation of the relation between the items of a given questionnaire. The obtained formal representation can be used to develop an adaptive and efficient tool for psychological assessment. An application of this methodology is presented with regard to the items of the Maudsley Obsessional-Compulsive Questionnaire (MOCQ). KST (Doignon \& Falmagne, 1985, 1999) has traditionally been applied for the efficient computerized assessment of knowledge and training (Doignon \& Falmagne, 1999; Dowling, Hockemeyer, \& Ludwig, 1996; Hockemeyer, Held, \& Albert, 1998); therefore, the main field of application of this theory is education. The computerized procedures built using this theoretical background provide evaluations, in the form of attitude outlines, showing what the subject is able to do and what he or she is ready to learn. One aim of this article is to identify how these useful aspects of KST can be applied to psychological assessment (e.g., clinical assessment, personality assessment, etc.). At least two main aspects have to be considered: (1) The main concepts of the theory have to be reinterpreted in light of this field of application, and it has to be verified whether this "translation" is allowed or not; (2) the theoretical hypothesized models have to be tested to determine whether they provide a good representation of the empirical data.
The main concepts of KST are described below. A knowledge domain can be defined as the set $Q$ of all the items that it is possible to investigate about a specific topic. The main authors of KST have focused their attention on topics included in the areas of mathematics and statistics, even if they did not exclude the possibility of applying the logical and formal structure of KST to different topics (Doignon \& Falmagne, 1999). Some further explanations will clarify both the reason why KST has been mostly applied in those specific contexts and why it has been used in this study.

Given the knowledge domain $Q$, a knowledge state $K \subseteq Q$ represents the subset of $Q$ that a specific subject is able to solve. A knowledge structure $\mathscr{K}$ is then defined as a collection of knowledge states including at least the empty set $(\varnothing)$ and the total set $(Q)$. In the traditional formal notation, a knowledge structure is denoted as $(Q, \mathscr{K})$, where $Q$ represents the knowledge domain and $\mathscr{K}$ represents the collection of subsets included in the structure. The knowledge structure is a representation of the implications among the items belonging to $Q$. Using this notation, it is possible to identify (1) the field of knowledge that is under consideration, (2) the specific knowledge presented by a single subject, and (3) the relations that link together the different items of $Q$.

An example may be useful for a better understanding of this last sentence. Consider the following knowledge structure defined for a set $Q$ of three items $a, b$, and $c$ :

A. Spoto, andrea.spoto@unipd.it; L. Stefanutti, luca.stefanutti@unipd.it; or G.Vidotto, giulio.vidotto@unipd.it 


$$
\mathscr{K}:=\{\varnothing,\{a\},\{b\},\{a, c\},\{a, b, c\}\} .
$$

In the knowledge structure $\mathscr{K}$, we can observe that the knowledge domain $Q$ is composed of the three items $a, b$, and $c$; the relations among the items in $Q$ determine the admissible knowledge states. In the given example, the mastery of item $a$ is a prerequisite for the mastery of item $c$ (i.e., $a$ is a prerequisite for $c$ ); in fact, there is no state in $\mathscr{K}$ containing $c$ and not containing $a$. In other words, any subject failing item $a$ would necessarily fail item $c$ (excluding the possibility of careless errors and lucky guesses).

When the collection $\mathscr{Y}$ of knowledge states of a knowledge structure $(Q, \mathscr{K})$ is closed under union (i.e., every union of knowledge states is again a knowledge state included in the structure; formally, $\cup \mathscr{F} \in \mathscr{K}$ for all $\mathscr{F} \subseteq \mathscr{K}$ ), the knowledge structure is then called a knowledge space.

An interesting property of a knowledge space is that more than a single set of prerequisites are allowed for an item. This means that the same item can be solved using different solution strategies (Doignon \& Falmagne, 1985).

Another fundamental concept of KST is that of a skill map (Albert, Schrepp, \& Held, 1992; Doignon \& Falmagne, 1999; Falmagne, Koppen, Villano, \& Doignon, 1990; Hockemeyer, Conlan, Wade, \& Albert, 2003; Lukas \& Albert, 1993). In our view, this concept can be easily adapted and reinterpreted in a clinical context. A skill map is a triple $(Q, S, f)$, where $Q$ is a nonempty set of items, $S$ is a nonempty set of skills, and $f$ is a mapping from $Q$ to $2^{S} \backslash\{\varnothing\}$ (i.e., the power set of $S$ excluding the empty set; Doignon \& Falmagne, 1999). For any item $q$ in $Q$, the subset $f(q)$ of $S$ represents the set of skills assigned to $q$. In other words, it means that if a subject solves item $q$, he or she has all the skills included in $f(q)$ (conjunctive model). In this article, we use the concept of a skill map and adapt it to the psychological assessment context in which the elements of $S$ are interpreted as clinical symptoms, rather than as abilities needed to solve a specific item.

In the Method section, a procedure for deriving a knowledge structure from a skill map is discussed. This procedure is essentially based on some interesting connections between KST and another theory, called FCA (Ganter \& Wille, 1999; Wille, 1982), pointed out by Rusch and Wille (1996).

Before starting the presentation of the specific aspects of the present study, it seems necessary to present some basic notions about FCA, in order to allow a better comprehension of its joint application with KST.

The first basic notion of FCA is the formal context, defined as a triple $(G, M, I)$, where $G$ is a set of objects, $M$ is a set of attributes, and $I$ is a binary relation between the set of objects and the set of attributes. A formal context is usually represented by a Boolean matrix in which each row is an object and each column is an attribute. Whenever a value 1 is present in the entry $(g, m)$, it means that the relation $\mathrm{gIm}$ holds; in other words, it means that the object $g$ has the attribute $m$. Between the objects and the attributes of a formal context, a Galois connection is defined. For all the sets $A \subseteq G$ and $B \subseteq M$, the following two transformations define the Galois connection:

$$
A^{\prime}:=\{m \in M \mid g I m, \forall g \in A\}
$$

and

$$
B^{\prime}:=\{g \in G \mid g I m, \forall m \in B\} .
$$

In words, $A^{\prime}$ is the collection of all the attributes that all the objects in $A$ have in common. Dually, $B^{\prime}$ is the collection of all the objects that possess all the attributes in $B$. It is now possible to introduce a fundamental notion of FCA. The pair $(A, B)$ is called a formal concept if it satisfies the following two conditions: $A=B^{\prime}$ and $B=A^{\prime}$. The so-called extent $A$ of the formal concept contains exactly those objects of $G$ that have all the attributes in $B$; the so-called intent $B$ of the formal concept includes exactly those attributes satisfied by all the objects in $A$.

A subconcept-superconcept relation is then defined in the following way:

$$
\left(A_{1}, B_{1}\right) \leq\left(A_{2}, B_{2}\right) \leftrightarrow A_{1} \subseteq A_{2}
$$

or equivalently,

$$
\left(A_{1}, B_{1}\right) \leq\left(A_{2}, B_{2}\right) \leftrightarrow B_{1} \supseteq B_{2} .
$$

In words, a concept is of a lower level when it has a larger extent (or equivalently, a smaller intent).

The concepts of a context form a complete lattice (Birkhoff, 1937, 1967), which is called the concept lattice of $(G, M, I)$. The intents of a concept lattice are closed under intersection; that is, each intersection of sets of attributes is included in the lattice. Rusch and Wille (1996) showed that the collection of the complements of the intents of a formal context is closed under set union, and so it is a knowledge space. In their article, the authors started from a formal context defined by the set $G$ of subjects (which, in this case, were treated as formal objects), the set $M$ of items, and the binary relation $\mathrm{gIm}$, meaning that the subject $g$ had correctly answered item $m$. In these terms, a response pattern can be seen as a set of formal attributes, an intent. With the relation between the intents of a formal context and their complements, the authors derived a socalled knowledge context having the domain defined by the set of items and the states by the complements of the observed response patterns. Using this methodology, it is then possible to construct a knowledge space starting from a formal context.

Concerning Point 2, introduced earlier in this section, an example of the joint application of KST and FCA in a clinical context, similar to the one introduced in Spoto, Vidotto, Postal, and Pendoni (2008), is presented. In this application, three knowledge structures representing the starting point for the construction of an adaptive computerized assessment tool are built and empirically tested. Such structures represent the relations between the items of the MOCQ (Hodgson \& Rachman, 1977) and the diagnostic criteria for obsessive-compulsive disorder (OCD) included in the Diagnostic and Statistical Manual of Mental Disorders-IV-TR (DSM-IV-TR; American Psychiatric Association [APA], 1995).

The results of this study have to be considered as a mere example of the potential use of the proposed approach. Furthermore, in the specific context, it is shown 
how the proposed methodology can be used as a tool for assessing a questionnaire's reliability and construct validity.

\section{METHOD}

\section{Structures Construction}

In this section, we briefly describe how existing FCA concept lattice construction algorithms have been applied for deriving the knowledge structure delineated by a given (conjunctive) skill map $(Q, S, f)$. A knowledge state $K$ is said to be delineated by a subset $X \subseteq S$ of skills via the conjunctive model if

$$
K=\{q \in Q: f(q) \subseteq X\} .
$$

The basic idea behind Equation 6 is that an item can be solved by a set $X$ of skills if all the skills $f(q)$ needed by that item are contained in $X$. Therefore, the collection of all items that are solvable by $X$ is the knowledge state $K$. Then, the resulting family of all such states is the knowledge structure delineated by $(Q, S, f)$ via the conjunctive model (Doignon \& Falmagne, 1999).

Two remarks are necessary. The first one regards the fact that the "skills" used in the present article are not considered in the sense of abilities needed to solve a particular subset of items, but in the sense of a set of diagnostic criteria satisfied by a patient who answers "true" to a particular subset of items. Therefore, the set $S$ contains diagnostic criteria rather than skills. The second remark refers to the use of the conjunctive model for depicting the relations among sets of attributes and set of items answered. In the conjunctive model, it is hypothesized that a subject who responds to an item would present all the attributes investigated by the item. Another possible approach to this issue is the disjunctive model (Doignon \& Falmagne, 1999). With this model, the answer to an item implies that the subject displays at least one of the attributes investigated by that item. It is easy to understand how, with this model, a different perspective is taken in looking at the clinician's interpretation of the score obtained by a patient.

It is now important to highlight a fundamental difference between the two models. With the conjunctive model, affirmative answers are more informative than negative ones. In fact, each affirmative answer indicates that the patient has all the attributes implied by the item. On the other hand, in the disjunctive model, negative answers are more informative than affirmative ones because they indicate that the patient does not have any attribute implied by the item. This consideration has important consequences on the practical side: It seems reasonable to use the conjunctive model when testing a clinical sample and a disjunctive model with a normal population. Although not done in this article, it would be interesting to assess the performance of the disjunctive model with the present data set.

The conjunctive model has been chosen because it represents the clinician's interpretation of the score obtained to a questionnaire, in the following sense. Usually, a clinician looks at the score obtained by the patient and evalu- ates whether it is clinically significant or not. In other words, he or she assumes that the patient displays all the characteristics investigated by the specific scale. Using the conjunctive model, it is possible to more deeply investigate the relations between the items on the basis of the attributes that each item satisfies, assuming that whenever a subject answers "true" to an item, he or she displays (or at least is supposed to possess) all the attributes investigated by that item.

The structure's construction algorithm rests on the following principles and concepts.

A formal context corresponding to $(Q, S, f)$ can be derived by interpreting $Q$ as the collection of objects and $S$ as the collection of attributes and by defining a binary relation $R \subseteq Q \times S$ so that, for all pairs, $(q, s) \in Q \times S$ :

$$
q R s \leftrightarrow s \notin f(q) .
$$

With these basic definitions at hand, the triple $(Q, S, R)$ can be regarded as a formal context. According to Equation 7, the notation $q R s$ should be read as skill s is not required by item $q$. As an effect of this definition, the intent $q^{\prime}:=\{s \in S \mid q R s\}$ is just the complement of $f(q)$ in $S$ (see, in this respect, Doignon \& Falmagne, 1999, p. 96). The collection $\mathscr{I}$ of all the intents of the concept lattice corresponding to this context could then be obtained by closing under intersection the collection $\left\{q^{\prime}: q \in Q\right\}$ of all object intents. There are a number of different algorithms doing this task in a quite efficient way (Ganter \& Wille, 1999, p. 64), and many different programs have been developed to implement these algorithms (Guénoche, 1990; Valtchev, Missaoui, Godin, \& Meridji, 2002; Vogt \& Wille, 1994). For any subset $X \subseteq S$, the corresponding extent $X^{\prime}$ is obtained by Equation 3. It is easily seen that by Equation 7, this collection can also be rewritten as

$$
X^{\prime}=\{q \in Q: f(q) \subseteq S \backslash X\},
$$

which, by Equations 6, happens to be a knowledge state delineated by $(Q, S, f)$. Then, it is a well-known fact in FCA that $X^{\prime \prime}$ (i.e., the set of attributes obtained by the application of Equation 3 to $\left.X^{\prime}\right) \in \mathscr{I}$ for all $X \subseteq S$. From this fact, it follows that, indeed, $\left\{Y^{\prime}: Y \in \mathscr{I}\right\}$ is the collection of all knowledge states delineated by the skill map at issue. To summarize, the construction procedure that has been used in practice was the following one: (1) After defining an appropriate skill map $(Q, S, f)$ for the items at hand, the relation $R$ corresponding to it was obtained by an application of the simple rule Equation 7; (2) then the whole concept lattice corresponding to the context $(Q, S, R)$ was produced by means of the software GaLícía (Valtchev et al., 2002); (3) at this point, the knowledge structure delineated by $f$ was simply the collection of all the extents of the generated concept lattice.

The knowledge structure composed by the extents of the concept lattice (i.e., sets of items) is closed under set intersection. It is known (Doignon \& Falmagne, 1985, 1999) that, in this case, each single item has a unique set of prerequisites. In the present article, and more generally in the analysis of a questionnaire, it is reasonable to accept this assumption. In fact, each item is defined by a set of 
attributes, and, via the conjunctive model, it is assumed that a person answering positively to an item has all the attributes of that item. Thus, the set of attributes is unique, and the minimal response pattern for each item is unique too. This consideration changes when applied to the whole diagnostic process, since it is not realistic to assume that a specific diagnosis is associated to a unique minimal set of symptoms.

\section{The Maudsley Obsessional-Compulsive Questionnaire}

The short form of the MOCQ (Sanavio \& Vidotto, 1985), included in the assessment battery Cognitive Behavioral Assessment 2.0 (Bertolotti, Zotti, Michielin, Vidotto, \& Sanavio, 1990), is composed of 21 dichotomous items (true-false) investigating the main characteristics of OCD. The questionnaire is subdivided into three subscales investigating three of the main specifications of the disorder.

The first subscale is called "Checking"; it is composed of eight items investigating some habits of controlling and recontrolling many things-for instance, Item 4, "I must check many times some particular things (e.g., gas or water taps, doors, etc.)," or Item 14, "I usually check things more than once." The score ranges from 0 to 8 , and the clinical cutoff is set at the 95th percentile.

The second subscale is called "Cleaning"; it is composed of nine items investigating the habits of washing, cleaning, and sense of contamination-for instance, Item 3, "When I touch an animal I feel contaminated," or Item 17, "Every morning I spend a lot of time in washing myself completely." The score ranges from 0 to 9 , and the clinical cutoff is set at the 95th percentile.

Finally, the third subscale is called "DoubtingRuminating"; it is composed of four items investigating the presence of intrusive and disagreeable thoughts - for instance, Item 2, "I frequently have disagreeable thoughts and I cannot get rid of them." The score ranges from 0 to 4 , and the clinical cutoff is set at the 95th percentile.

The items of the MOCQ-R mostly reflect the diagnostic criteria for OCD included in the DSM. In order to analyze the items of the MOCQ-R, we used the latest version of the manual, the DSM-IV-TR (APA, 1995). OCD is included in the category of "Anxiety Disorders," and its diagnosis is based on five main criteria. The first criterion has been further subdivided into two main parts: The first one deals with "Obsessions," and the second one refers to "Compulsions."

In the present article, the diagnostic criteria of DSMIV-TR have been used to analyze each single item of the three scales of the MOCQ-R. The result of such analysis is represented by three formal contexts (consisting of three Boolean matrices), where the objects of the context are the items of the subscale and the attributes are the diagnostic criteria of the DSM-IV-TR for OCD. The underlying relation $(\mathrm{gIm})$ indicates that the item $g$ investigates the criterion $m$. In the next subsection, the details of the procedure are described. In particular, the method of construction of the structures is described, and some descrip- tive indications about the attributes' implications revealed by the structures are provided.

\section{The Structures of the MOCQ-R}

In Figure 1, the concept lattice (i.e., the knowledge structure having sets of items as states) obtained for the "Doubting-Ruminating" subscale is presented.

A short explanation of this kind of figure is needed. Figure 1 displays the complete lattice obtained for the "Doubting-Ruminating" scale. Each node of the lattice represents a formal concept. The number assigned to each node is not important (in this article). In the present article, we are interested in the collection of objects (items) and attributes (diagnostic criteria) listed in each single gray rectangle. The prerequisite relation among items has to be read bottom-up in the figure. These general rules have to be applied to all the figures included in this article.

Going into the details of Figure 1, it is interesting to note that there are three different paths from the empty set to the total set of items. More specifically, from the structure it emerges how Item 5 is a prerequisite for Item 2 ; in fact, there is no state including Item 2 and not including Item 5. From the attributes point of view, it appears that Item 2 has all the attributes of Item 5 plus some other attributes. In Figure 1, it seems that the intent of the set of objects $\{5,2\}$ (see Node 2 in Figure 1) contains fewer elements (i.e., B, Cb, CA1a, Ca, CA2a, CA2b, CA1c, Cd) than does the intent of Item 5 (see Node 4 in Figure 1; i.e., B, Cb, CA1a, Ca, CA2a, CA2b, CA1c, Cd, OA1b, OA3). In fact, the structure of Figure 1 is derived by the dual of the formal context having the four items of the "Doubting-Ruminating" subscale as objects and the diagnostic criteria of DSM-IV-TR as attributes; that is, the attributes included in Nodes 2 and 4 are not satisfied, respectively, by the sets of items $\{5,2\}$ and $\{5\}$.

In Figure 2, the concept lattice obtained for the "Checking" subscale is presented.

Given this structure, it seems that Item 15 ("I follow a very precise order in everything I do") is a prerequisite for most items, and this indicates that the attributes present in it are replicated in many other items of the subscale.

The remarks on the "Cleaning" structure will be presented later in the Results section, because some further elements have to be introduced in order to understand the steps followed to derive the final structure.

\section{Testing the Structures}

Knowledge structures like the ones presented above are, by definition, deterministic; they represent a model of possible response patterns of a sample of subjects, but they do not predict the probability of each pattern. As has been suggested by Doignon and Falmagne (1999), there are two main reasons to introduce probabilities into the model: The first one is that each state should be present with different frequencies in the population; the second one is that the observed response pattern of a subject could not represent his/her real knowledge. From the second reason, the opportunity to consider two parameters related to each item follows: the careless error (also called 


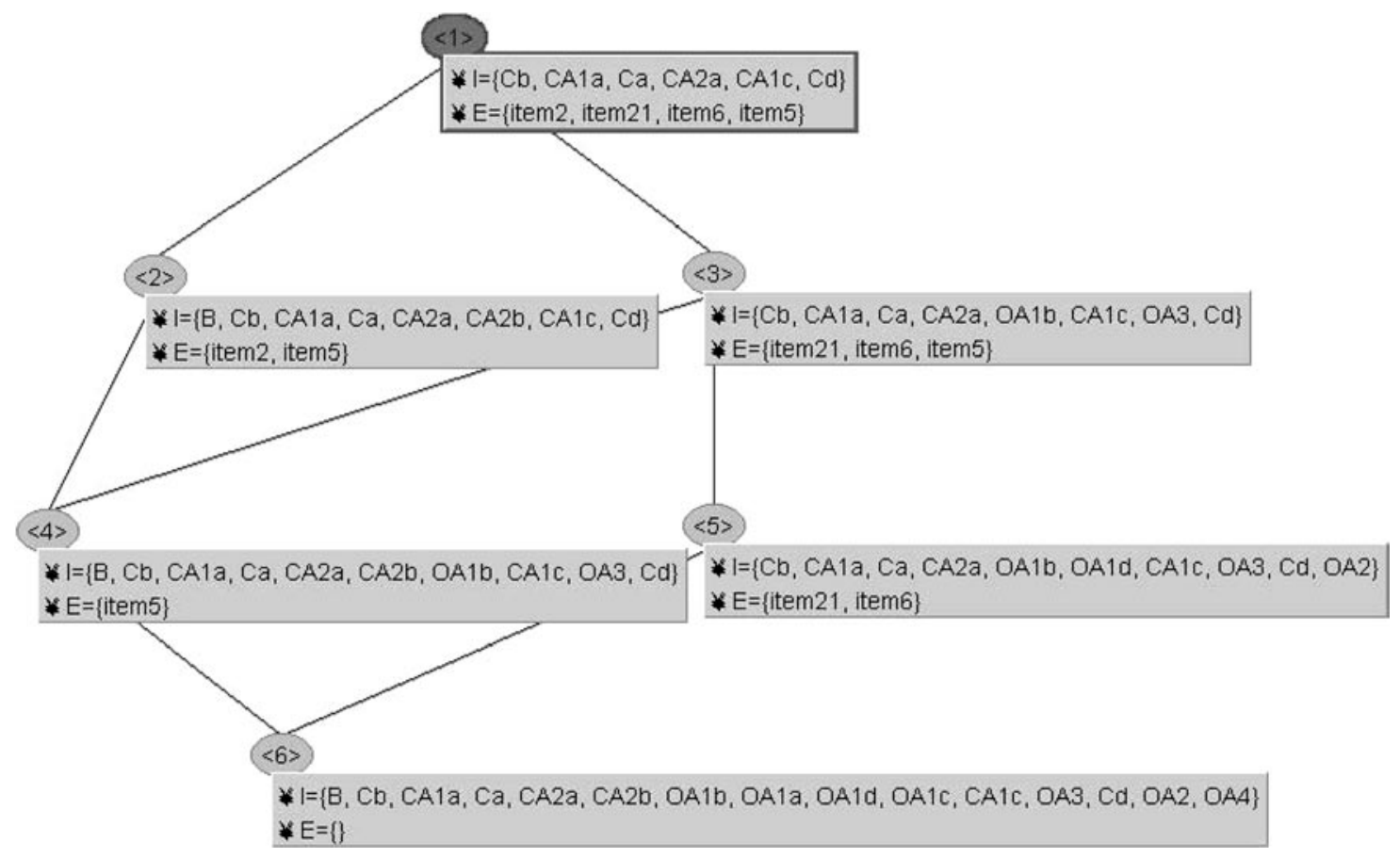

Figure 1. The concept lattice obtained for the "Doubting-Ruminating" subscale.

false negative; $\alpha$ ) and the lucky guess (also called false positive; $\beta$ ) represent, respectively, the probability that a subject does not solve an item that he or she is able to solve and the probability of solving an item that he or she is not able to solve. In other words, it is reasonable to introduce conditional probabilities of responses, given the knowledge states.

Falmagne and Doignon (1988a) define a probabilistic knowledge structure as a triple $(Q, \mathscr{K}, p)$, where $(1)(Q, \mathscr{K})$ is a knowledge structure, and (2) $p$ is a probability distribution for $\mathscr{K}$. In the model at issue, given a state, the responses to the items are locally independent. Thus, starting from the probabilistic knowledge structure $(Q, \mathscr{K}, p)$, given a specific response pattern $R \subseteq Q$, we will define a function $s:(R, K) \mapsto s(R, K)$, assigning to each response pattern its conditional probability given that a subject is in state $K$ (for all states $K \in \mathscr{K}$ ), the response function for the probabilistic knowledge structure. Thus, we obtain for each response pattern a probability distribution:

$$
p(R)=\sum_{K \in \mathscr{K}} s(R, K) p(K) .
$$

Since the response function $s$ satisfies local independence for each item $q \in Q$, the conditional probability $s(R, K)$ is determined given the two probabilities $\alpha$ and $\beta$, the careless error and lucky guess, respectively, related to each item $q$. Formally,

$$
\begin{aligned}
s(R, Q)= & {\left[\left(\prod_{q \in K \backslash R} \alpha_{q}\right)\left(\prod_{q \in K \cap R}\left(1-\alpha_{q}\right)\right]\right.} \\
& \cdot\left[\left(\prod_{q \in R \backslash K} \beta_{q}\right)\left(\prod_{q \in \overline{R \cup K}}\left(1-\beta_{q}\right)\right] .\right.
\end{aligned}
$$

Equation 10 represents the "basic local independence model" (BLIM), which is used in the present article.

The parameters of the model have been estimated by the expectation-maximization algorithm (Dempster, Laird, \& Rubin, 1977).

\section{RESULTS}

In order to validate the obtained structures, a data set provided by a sample of patients from the northeastern part of Italy ( $n=33$; ages ranging from 19 to 43 years; 20 males, 13 females) with a diagnosis of OCD has been used. The parameters of the BLIM were estimated for each of the three structures. The fit of each of the three models was tested by Pearson's chi-square. It is well known that for large data matrices (such as those used in the present study), the asymptotic distribution of $\chi^{2}$ is not reliable. Therefore a $p$ value for $\chi^{2}$ has been obtained by parametric bootstrap (number of replications $=5,000$ ).

In the first part of the analysis we tested, for the "Cleaning" subscale (nine items), a knowledge structure composed of 80 knowledge states derived by the closure under intersection of the intents of the formal context. The single items' $\alpha$ and $\beta$ parameters seem quite small for almost all items (Table 1).

As was previously discussed, in KST, $\alpha$ and $\beta$ represent the probability of careless errors and lucky guesses, respectively. It seems more appropriate, in the present article, to refer to them as false negative and false positive in their clinical acceptation.

The results of the bootstrap performed on this model do not support the goodness of fit of the structure $\left(\chi^{2}=\right.$ $179.98, p=.0487)$. By the analysis of the content of each 


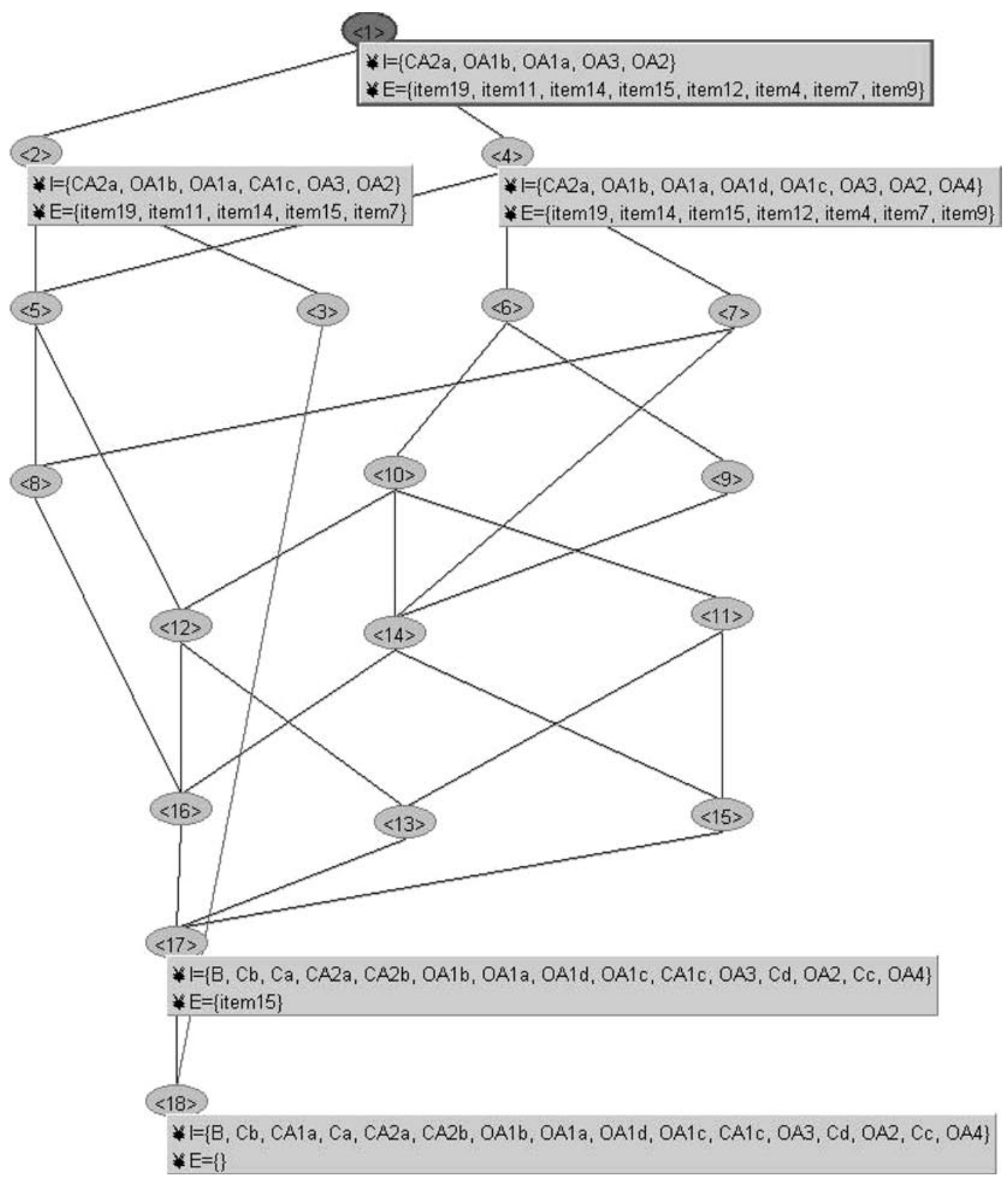

Figure 2. The concept lattice obtained for the "Checking" subscale.

single item, the deletion of Item 1 appears to be the best solution to the poor fit. In fact, it says, "I do not use the public phone because I am afraid of possible contaminations," which seems rather obsolete. The deletion of this item reduces the number of possible states to 40 .

The new structure (i.e., without Item 1) has been tested together with the structures obtained for the other two subscales. The results show good fit indexes for all three models. Table 2 displays the global fit indexes obtained for the three models, along with corresponding $p$ values obtained by parametric bootstrap.

The $p$ value of the bootstrap performed on the "Cleaning" subscale needs some further explanation. The small value observed could be explained by the fact that the number of states derived by closure under intersection of the formal context is about 40 . This is due to the fact that in this subscale, the items are rather heterogeneous. They investigate a number of attributes higher than the one investigated by the items of the other subscales. The number of states found for the "Doubting-Ruminating" scale is 6 , and the number of states found for the "Checking" subscale is 19. A larger sample could improve the level of the $p$ value also, because the levels of $\alpha$ and $\beta$ are good, especially for the "Cleaning" subscale. Table 3 shows the $\alpha$ and $\beta$ parameters obtained for each item in the test.

The overall model fit, along with the results displayed in Table 3, indicates that the three models quite accurately depict the relations between different items of the 
Table 1

Estimated Parameters $\alpha$ and $\beta$ for Each Item of the "Cleaning" Scale

\begin{tabular}{cccccccccc}
\hline & \multicolumn{7}{c}{ Item } \\
\cline { 2 - 10 } Parameter & 1 & 3 & 8 & 10 & 13 & 16 & 17 & 18 & 20 \\
\hline$\alpha$ & .00 & .26 & .11 & .00 & .25 & .05 & .00 & .16 & .00 \\
$\beta$ & .00 & .00 & .00 & .31 & .35 & .00 & .00 & .00 & .00 \\
\hline
\end{tabular}

Table 2

The Global Fit Indexes of the Three Models

\begin{tabular}{lcrrr}
\hline \multicolumn{1}{c}{ Model } & No. of & & & \\
& States & \multicolumn{1}{c}{$d f$} & \multicolumn{1}{c}{$\chi^{2}$} & Bootstrap $p$ \\
\hline Checking & 18 & 222 & 127.39 & .2186 \\
Cleaning & 42 & 198 & 141.65 & .1003 \\
Doubting-ruminating & 6 & 2 & 2.30 & .8056 \\
\hline
\end{tabular}

MOCQ-R. These relations are well represented by the formal context built using the items of MOCQ-R as objects and the diagnostic criteria of DSM-IV-TR for OCD as attributes. The two critical parameters - that is, the high values of false positive estimates for Items 2 and 12 - can be explained in two different but not necessarily exclusive ways. The first one refers to the small number of subjects composing the sample. The second one relates to the fact that the items are clinical; thus, a sort of misinterpretation of the meaning of the items is possible. This fact can be better understood by looking at the text of the items. Item 2 says, "I frequently have disagreeable thoughts and I cannot get rid of them." This item is composed of two separate sentences: The first one is "I frequently have disagreeable thoughts"; the second one is "I cannot get rid of them." In the conjunctive model, a subject is supposed to provide a positive answer when he has all the elements required. In this case, the subject may have answered "true" even if he believed that only one of the two sentences was true. Item 12 says, "One of the greatest problems of mine is the repeated check of things." In this case, some problems may arise from the interpretation of either "greatest" or "repeated." Anyway, since the analyzed questionnaire is clinical, it seems reasonable to expect higher $\alpha$ and $\beta$ values than in the classical field of application of KST. In fact, a subject could intentionally fake the answer. Furthermore, the subject's answer could be affected by his poor introspection capabilities.

\section{DISCUSSION}

The present article provides some interesting results from both the clinical and the methodological point of view.

From the methodological point of view, the proposed analysis can be used as a tool to validate the construct and content validity of a given questionnaire. In the presented example, the validity of MOCQ-R has been assessed on the basis of the presence/absence of the criteria of the DSM-IV-TR, which gives a theoretical-clinical interpretation of the questionnaire. The joint application of FCA and KST can evaluate whether a questionnaire actually measures the underlying construct (in this case, OCD). In the example, the underlying construct was the set of diagnostic criteria for OCD. In this perspective, the validity analysis rests on verifying the relation between the items and the criteria (i.e., the attributes of the formal context).

In typical applications, a clinician uses the questionnaire by considering the score obtained by the patient. Since the underlying construct is multidimensional, the mere score of a patient is a dramatic reduction of the potential information provided by the test. By the proposed approach, the information collected by the questionnaire can be used to point out differences between patients that otherwise would be hidden by the simple score. Indeed, from the clinical perspective, the proposed methodology could be regarded as an in-depth evaluation of the construct investigated by the questionnaire. The response patterns corresponding to clinically significant scores (i.e., $>95$ th percentile) could point to different collections of diagnostic symptoms, and these differences are not captured by the simple score. For instance, it is possible to note that the two collections of items $\{3,8,10,13,16,18,20\}$ and $\{3,8,13,16,17,18$, $20\}$ (corresponding to Nodes 2 and 4 in Figure 3, respectively) are equally scored 7 but they correspond to different collections of attributes. This means that patients obtaining the same score might have different sets of symptoms.

In the presented case, the relation between the items of the MOCQ-R and the criteria of the DSM-IV-TR has been constructed assuming a conjunctive model. As was discussed in the Structure Construction section, this assumption seems reasonable when a sample of clinical patients is assessed. There are other situations in which the disjunctive model could be more appropriate-for instance, when reasoning in terms of a whole diagnostic process in a wider spectrum situation (i.e., a complete battery for clinical assessment).

Considering the practical application of the approach proposed in this article, in a clinical setting, the relations found between items could be used to calibrate an algorithm for the adaptive and efficient evaluation of patients. KST has been developed with the aim of constructing an efficient tool for assessing knowledge. Some computerized algorithms have been developed with this aim (Dowling et al., 1996; Falmagne \& Doignon, 1988a, 1988b; Hockemeyer et al., 1998). These algorithms can be easily adapted to the clinical context for which the structures of Figures 1, 2, and 3 have been developed.

Table 3

Estimated Parameters $\alpha$ and $\beta$ for Each Item of the Three Subscales

\begin{tabular}{|c|c|c|c|c|c|c|c|c|}
\hline \multicolumn{3}{|c|}{ Checking } & \multicolumn{3}{|c|}{ Cleaning } & \multicolumn{3}{|c|}{$\begin{array}{l}\text { Doubting- } \\
\text { Ruminating }\end{array}$} \\
\hline Item & $\alpha$ & $\beta$ & Item & $\alpha$ & $\beta$ & Item & $\alpha$ & $\beta$ \\
\hline 4 & .00 & .00 & 3 & .27 & .07 & 2 & .00 & .40 \\
\hline 7 & .24 & .23 & 8 & .21 & .00 & 5 & .06 & .00 \\
\hline 9 & .06 & .13 & 10 & .00 & .21 & 6 & .22 & .00 \\
\hline 11 & .00 & .26 & 13 & .23 & .27 & 21 & .10 & .09 \\
\hline 12 & .00 & .40 & 16 & .05 & .00 & & & \\
\hline 14 & .00 & .00 & 17 & .00 & .00 & & & \\
\hline 15 & .16 & .00 & 18 & .24 & .00 & & & \\
\hline 19 & .00 & .20 & 20 & .16 & .00 & & & \\
\hline
\end{tabular}




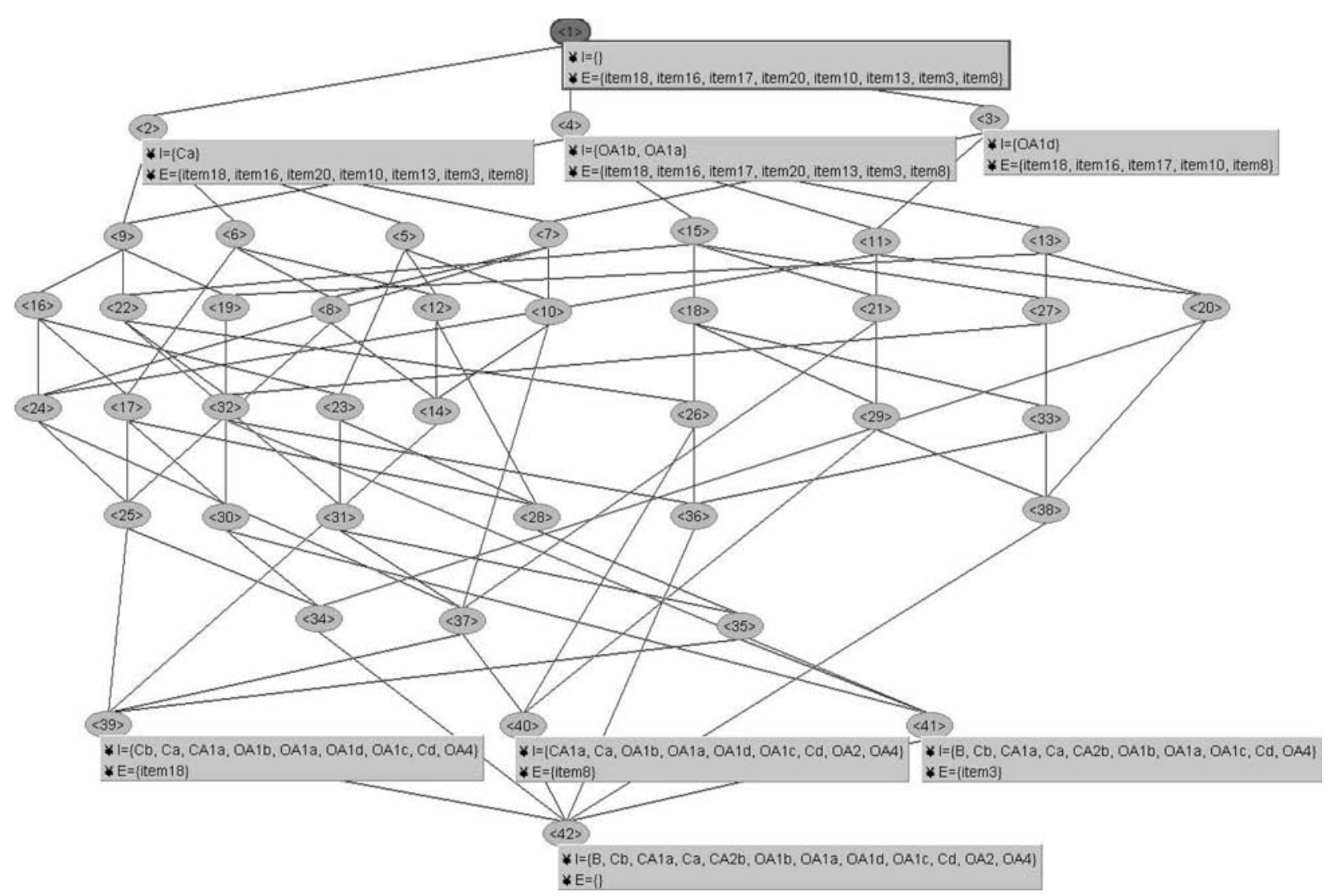

Figure 3. The concept lattice obtained for the "Cleaning" subscale, excluding Item 1.

A final remark concerns the $\alpha$ and $\beta$ probabilities of the items. Since they are interpreted as false positives and false negatives, these probabilities are clearly expected to be small. Large values of these parameters could point to bad specification of the model or to bad wording of the items. Therefore, these parameters can be used as a diagnostic tool for improving the model or the items. For instance, when a large value of the parameter $\alpha$ is observed for a given item, there might be one or more attributes both necessary to positively answer to a specific item and not included in the model for that item. In other words, even if, in a given model, the attributes referred to a specific item $i$ are, for instance, $\{a, b, c\}$, a large value of $\alpha$ indicates that one or more further attributes have to be displayed by a subject to answer $i$. There are two main ways to cope with this problem: The first one is to reformulate the model with respect to $i$, including the necessary attributes; the second one is to reword the specific item so that it is in accord with the model. Working with standardized questionnaires, the first solution might be preferred; but when a questionnaire is under construction, the second approach could be the most interesting one.

Some different issues have to be considered when coping with a large value of $\beta$. There are two main explanations of this. The first one is that in the model, one or more attributes have been wrongly considered necessary for answering "true" to a specific item. In this case, it is possible to eliminate from the set of the attributes of that item all those attributes that are not necessary for it; otherwise, an alternative could be the rewording of the item. The second explanation is that the conjunctive model is not a good representation of the relation between items and attributes. In this case, the possibility of applying a disjunctive model can be considered.

In conclusion, the present article highlights how the proposed application to a clinical context of KST and FCA seems to be a useful and fruitful research and clinical perspective. It has to be further investigated (using larger samples and different clinical disorders) whether the proposed methodology would be able to provide the clinician with the opportunity of performing a more indepth analysis of patients' responses. This opportunity would allow the clinician to do personalized diagnosis able to pinpoint subject-specific characteristics. In the long term, this methodology will allow the construction of an adaptive, efficient, and effective tool for psychological diagnosis. This instrument will be very similar to the instruments already built for knowledge assessment.

\section{AUTHOR NOTE}

The authors thank Ezio Sanavio and the Institute of Cognitive and Behavioral Therapy of Padua for providing the questionnaires filled out by OCD patients and for giving many useful suggestions. The authors also thank Luigi Burigana and Eraldo Nicotra for their useful suggestions. Correspondence concerning this article should be addressed to A. Spoto, L. Stefanutti, or G. Vidotto, Department of General Psychology, Univer- 
sity of Padua, Via Venezia 8, 35131 Padua, Italy (e-mail: andrea.spoto@ unipd.it, luca.stefanutti@unipd.it,or giulio.vidotto@unipd.it).

\section{REFERENCES}

Albert, D., Schrepp, M., \& Held, T. (1992). Construction of knowledge spaces for problem solving in chess. In G. H. Fisher \& D. Laming (Eds.), Contributions to mathematical psychology, psychometrics, and methodology (pp. 123-135). New York: Springer.

American Psychiatric Association (1995). DSM-IV-TR: Diagnostic and statistical manual of mental disorders (4th ed., text revision). Washington, DC: Author.

Bertolotti, G., Zotti, A. M., Michielin, P., Vidotto, G., \& SANAVIO, E. (1990). A computerized approach to cognitive behavioural assessment: An introduction to CBA-2.0 primary scales. Journal of Behavior Therapy \& Experimental Psychiatry, 21, 21-27.

BIRKHOFF, G. (1937). Rings of sets. Duke Mathematical Journal, 3, 443454.

BIRKHoff, G. (1967). Lattice theory. Providence, RI: American Mathematical Society Colloquium Publication.

Dempster, A. P., Laird, N. M., \& Rubin, D. B. (1977). Maximum likelihood from incomplete data via the EM algorithm. Journal of the Royal Statistical Society B, 39, 1-38.

Doignon, J. P., \& Falmagne, J. C. (1985). Spaces for the assessment of knowledge. International Journal of Man-Machine Studies, 23, 175-196.

Doignon, J. P., \& Falmagne, J. C. (1999). Knowledge spaces. Berlin: Springer.

Dowling, C. E., Hockemeyer, C., \& Ludwig, A. H. (1996). Adaptive assessment and training using the neighbourhood of knowledge spaces. In C. Frasson, G. Gauthier, \& A. Lesgold (Eds.), Intelligent tutoring systems (pp. 578-586). Berlin: Springer.

Falmagne, J. C., \& Doignon, J. P. (1988a). A class of stochastic procedures for the assessment of knowledge. British Journal of Mathematical \& Statistical Psychology, 41, 1-23.

Falmagne, J. C., \& Doignon, J. P. (1988b). A Markovian procedure for assessing the state of a system. Journal of Mathematical Psychology, 32, 232-258.

Falmagne, J. C., Koppen, M., Villano, M., \& Doignon, J. P. (1990). Introduction to knowledge spaces: How to build, test and search them. Psychological Review, 97, 201-224.
GANTER, B., \& Wille, R. (1999). Formal concept analysis: Mathematical foundations. Berlin: Springer.

Guénoche, A. (1990). Construction du treillis de Galois d'une relation binaire. Mathmatiques et Sciences Humaines, 109, 41-53.

Hockemeyer, C., Conlan, O., Wade, V., \& Albert, D. (2003). Applying competence prerequisite structures for e-learning and skill management. Journal of Universal Computer Science, 9, 1428-1436.

Hockemeyer, C., Held, T., \& Albert, D. (1998, June). Rath: A relational adaptive tutoring hypertext www-environment based on knowledge space theory. Paper presented at the CALISCE '98 meeting (Computer Aided Learning in Science and Engineering). Göteborg, Sweden.

Hodgson, R. J., \& Rachman, S. J. (1977). Obsessional-compulsive complaints. Behaviour Research \& Therapy, 15, 389-395.

LuKas, J., \& AlberT, D. (1993). Knowledge assessment based on skill assignment and psychological task analysis. In G. Strube \& K. F. Wender (Eds.), The cognitive psychology of knowledge (pp. 139-159). Amsterdam: Elsevier.

Rusch, A., \& Wille, R. (1996). Knowledge spaces and formal concept analysis. In H. H. Bock \& W. Polasek (Eds.), Data analysis and information systems: Statistical and conceptual approaches (pp. 427-436). Berlin: Springer.

Sanavio, E., \& Vidotto, G. (1985). The components of the Maudsley Obsessional-Compulsive Questionnaire. Behaviour Research \& Therapy, 23, 659-662.

Spoto, A., Vidotto, G., Postal, D., \& Pendoni, E. (2008). Rappresentazione formale dell'assessment cognitvo-comportamentale per il disturbo ossessivo-compulsivo. Giornale Italiano di Medicina del Lavoro ed Ergonomia: Supplemento B. Psicologia, 30, B91-B100.

Valtchev, P., Missaoui, R., Godin, R., \& Meridji, M. (2002). Generating frequent itemsets incrementally: Two novel approaches based on Galois lattice theory. Journal of Experimental \& Theoretical Artificial Intelligence, 14, 115-142.

Vogt, F., \& Wille, R. (1994). Toscana: A graphical tool for analyzing and exploring data. In R. Tamassia \& I. G. Tollis (Eds.), Graph drawing (pp. 226-233). New York: Springer.

Wille, R. (1982). Restructuring lattice theory: An approach based on hierarchies of concepts. In I. Rival (Ed.), Ordered sets (pp. 445-470). Dordrecht: Reidel.

(Manuscript received June 26, 2009; revision accepted for publication September 20, 2009.) 